\title{
Forecasting the size and peak of cholera epidemic in Yemen, 2017
}

\author{
Hiroshi Nishiura*,1, Shinya Tsuzuki ${ }^{1}$ \& Yusuke Asai ${ }^{1}$ \\ ${ }^{1}$ Graduate School of Medicine, Hokkaido University, Kita 15 Jo Nishi 7 Chome, Kitaku, Sapporo, 060-8638, Japan \\ * Author for correspondence: nishiurah@med.hokudai.ac.jp
}

\begin{abstract}
' $T$ The predictive performance of mathematical models has been demonstrated through empirical studies of spatiotemporal epidemic prediction, in particular, via global forecasting exercises of emerging infectious diseases"
\end{abstract}

First draft submitted: 20 October 2017; Accepted for publication: 27 October 2017; Published online: 21 February 2018

Keywords: cholera $\bullet$ epidemic $\bullet$ forecasting $\bullet$ mathematical model $\bullet$ prediction $\bullet$ statistical estimation

\section{Forecasting tools of infectious disease epidemics}

Epidemic models are increasingly used for forecasting purposes. The predictive performance of mathematical models has been demonstrated through empirical studies of spatiotemporal epidemic prediction, in particular, via global forecasting exercises of emerging infectious diseases [1,2]. Such models are represented by those applied to SARS, influenza A (H1N1-2009) and Middle East respiratory syndrome [1]. The success of global forecasting has been partly supported by recent advances in computational statistics, especially those employing the Markov Chain Monte Carlo technique. Additionally, the epidemiological dynamics of global spread has been shown to be mainly (and mostly) characterized by the global mobility pattern of humans [3]. The time from emergence of a novel infectious disease in a country to arrival at an importing country is linearly predicted by using a simple metric of the airline transportation network (which is referred to as the 'effective distance'). This has not only enabled us to exploit the metric for real-time forecasting of global spread of Middle East respiratory syndrome and Zika virus infection [4,5], but also led us to analyze the effectiveness of travel restriction policies in preventing international spread of Ebola virus disease [6].

In addition to the progress in forecasting theory and techniques, epidemiological models have been considerably improved to capture actual transmission dynamics using epidemiological determinants of transmission as an additional input variable (in addition to patient data from surveillance). For instance, the dependence of influenza transmission on humidity has been empirically demonstrated by adding humidity as an explanatory variable of the transmission coefficient to the so-called susceptible-infectious-recovered model [7]. In this instance, humidity acts as an input variable of part of the transmission model parameters, and it is pleasing that both mechanistic understanding of the epidemic dynamics and predictive performance are jointly improved only by replacing a constant transmission coefficient by a simple mathematical function of humidity.

Promising mechanistic models for forecasting would require additional data to surveillance information in order to ensure improved performance. However, airline transportation network data and spatiotemporal data of humidity are both regarded as part of the big data, and their incorporation into epidemic modeling would require substantial effort and computational resources for data collection and analysis. It is often practically the case that the input data are very limited, especially in cases where the epidemic is in a country where the civil war is underway. Forecasting in a data-limited setting requires a more parsimonious modeling approach. Here we describe how we overcame the limitation of input data during the cholera epidemic in Yemen, 2017 [8].

\section{Cholera in Yemen}

Cholera is a bacterial infection caused by Vibrio cholerae. Through contaminated water or an environmental object, the bacterium infects the human small intestine, preventing infected individuals from properly digesting and absorbing water from the intestinal tract. Infected individuals experience diarrhea of various types, vomiting and

Future $\because$ Medicine 
dehydration. A large cholera epidemic caused by serotype Ogawa strain has been observed in Yemen, a Middle East country since 2016. While the epidemic was clearly in decline in early 2017, a drastic resurgence has been observed since April 2017. As of 1 October 2017, a total of 777,229 cases have been reported since April 2017, among which there were 2134 mortalities. The size of the outbreak is unprecedented.

Given the massive number of cases, it is unlikely that a forecasting model can capture the substantial heterogeneities based on individual-level data, while yielding a valid quantitative forecast. The dataset that we had an access to was an epidemic curve that has been updated every week. The so-called generalized logistic model, one of phenomenological models, involves only four unknown parameters and is recognized to be able to capture a unimodal curve flexibly $[8,9]$ :

$$
J(t)=\frac{K}{\left[1+g \exp \left(-r\left(t-t_{i}\right)\right)\right]^{\frac{1}{g}}},
$$

(Equation 1).

where $J(t)$ is the cumulative weekly number of cases, and thus, the weekly incidence is described by the difference of $J(t)$ by 1 week:

$$
j_{t}=J(t)-J(t-1)
$$

(Equation 2).

Parameter $K$ is referred to as the carrying capacity, representing the cumulative incidence at time infinity, that is, our interest (or the ultimate size of the epidemic). Parameter $r$ is the growth rate, $t_{\mathrm{i}}$ is the point of inflection, corresponding to the peak timing of an epidemic, and $g$ is the parameter that partially determines the point of inflection on the vertical axis.

\section{Addressing reporting delay \& under ascertainment}

Not only is there limited amounts and types of data, but Yemen data also involve two epidemiological problems. First, an epidemic curve is continuously reported by the WHO, but the past weekly incidence data have to be continuously updated. For example, the number of cases in the latest weeks would likely be revised in the future, and the count is mainly affected by the delay from illness onset to diagnosis and reporting (i.e., the so-called 'reporting delay'). To offer accurate real-time forecast, such reporting delays need to be incorporated into the prediction model. Therefore, let $j_{\mathrm{t}}$ be the actual (unbiased) incidence in week $t$. That is, we explicitly distinguish reported incidence $c$ from the actual incidence $j$. Let $F_{\Delta \mathrm{t}}$ be the cumulative distribution function of the time from illness onset to reporting as a function of the reporting delay $\Delta t$ days. The expected value of the weekly reported incidence is described as:

$$
E\left[c_{t, \Delta t}^{\prime}\right]=j_{t} F_{7(s-t)+\Delta t},
$$

(Equation 3).

where $s$ represents the latest week of observation (for $t \leq s$ ).

Second, once a large epidemic is recognized, it is expected that the chance of diagnosis and reporting would increase. That is, physicians and healthcare workers may doubt a cholera diagnosis or be reluctant to report suspected cholera during the very early stage of the epidemic, but as the epidemic is increasingly recognized, such underascertainment is drastically improved. Such ascertainment bias is reflected in the estimate of the weekly ratio of deaths to cases (i.e., the case fatality risk). Furthermore, the ratio of weekly number of deaths to that of cases in late April has been more than 7.3-times greater than the ratio in early July 2017. The time-dependent decrease in the ratio of deaths to cases should reflect improved ascertainment. Let the relative case fatality risk of week $t$ be $\alpha_{\mathrm{t}}$. The expected value of the weekly incidence is further improved and written as:

$$
E\left[c_{t, \Delta t}\right]=\frac{j_{t}}{\alpha_{t}} F_{7(s-t)+\Delta t} .
$$

(Equation 4).

To our knowledge, the above-mentioned equation for cholera was the first to implement real-time forecasting [8] while jointly addressing reporting delay and ascertainment bias. 


\section{Estimated week of epidemic peak \& size of cholera in Yemen}

Employing the above-mentioned model and the data up to week 26, 2017, the forecast was obtained [8]. The cumulative incidence was estimated by using the carrying capacity as a parameter, and its real-time estimate was 790,778 (95\% CI: 700,495-914,442) cases and 767,029 (95\% CI: 690,877-871,671) cases, respectively, by using logistic and generalized logistic models. It was also indicated that the epidemic peak has passed by week 26, 2017, and thus, the weekly incidence was predicted to decrease from week 27 onwards [8].

It is $>10$ weeks since the estimation was carried out. The largest incidence was actually observed in week 26 , 2017. The epidemic took the path of clearly decreasing trend, but the speed of actual decline appeared to be slower than predicted. As such, the epidemic size is likely to be greater than what it was predicted in week 26, 2017, potentially reaching to 1 million cases.

The underestimation of the epidemic size is qualitatively attributable to the mismatch between logistic-family model and the actual slow decline in weekly incidence after week 26. Such slow decline could be generated by heterogeneities that are attributable to geographic reasons or other nonhomogeneities in the transmission. Unfortunately, the flexible curve can address varying shapes of unimodal curve alone and cannot capture small resurgences that result in a multimodal shape. However, qualitatively the shape of an epidemic curve and peak timing has been most appropriately captured.

\section{Conclusion \& future perspective}

The peak timing is certainly recognizable when the time is close to the epidemic peak [10], as endorsed by the cholera data in Yemen. Two important different directions for future improvement must be emphasized. First, the phenomenological model could potentially be extended to heterogeneous settings, for example, geographically structured data. Analysis of multiple spatial dimensions could help reduce uncertainty as compared with an analysis of single epidemic curve. An improved function that captures spatial interaction (i.e., geographic dependence) within the phenomenological model is called for, and such a model should be able to capture the recent small resurgence of cases in week 34, 2017, Yemen.

Second, a semi-mechanistic approach with conditional fitting may be better compared against fitting a single functional curve (i.e., logistic model) to the data. Since a fully mechanistic model can never capture the actual transmission dynamics in empirical settings, a greatly compromised mechanistic model that may even not be distinguishable from phenomenological models would be required for improved prediction accuracy. If a semimechanistic model with conditional fitting procedure is employed, this would not only capture a small part of the epidemic mechanisms, but also stochastic dependence structure would be addressed.

Financial \& competing interests disclosure

$\mathrm{H}$ Nishiura received funding support from the Japan Agency for Medical Research and Development and the Japan Science and Technology Agency CREST program (JPMJCR1413) and RISTEX program for Science of Science, Technology and Innovation Policy, the Japan Society for the Promotion of Science KAKENHI 16KT0130 and 17H04701. The funders had no role in study design, data collection and analysis, decision to publish or preparation of the manuscript. The authors have no other relevant affiliations or financial involvement with any organization or entity with a financial interest in or financial conflict with the subject matter or materials discussed in the manuscript apart from those disclosed.

No writing assistance was utilized in the production of this manuscript.

\section{Open access}

This work is licensed under the Attribution-NonCommercial-NoDerivatives 4.0 Unported License. To view a copy of this license, visit http://creativecommons.org/licenses/by-nc-nd/4.0/

\section{References}

1 Colizza V, Barrat A, Barthélemy M et al. The modeling of global epidemics: stochastic dynamics and predictability. Bull. Math. Biol. 68(8), 1893-1921 (2006).

2 Van den Broeck W, Gioannini C, Gonçalves B et al. The GLEaMviz computational tool, a publicly available software to explore realistic epidemic spreading scenarios at the global scale. BMC Infect. Dis. 11, 37 (2011).

3 Brockmann D, Helbing D. The hidden geometry of complex, network-driven contagion phenomena. Science 342(6164), 1337-1342 (2013). 
4 Nah K, Mizumoto K, Miyamatsu Y et al. Estimating risks of importation and local transmission of Zika virus infection. PeerJ 4, e1904 (2016).

5 Nah K, Otsuki S, Chowell G et al. Predicting the international spread of Middle East respiratory syndrome (MERS). BMC Infect. Dis. 16,356 (2016).

6 Otsuki S, Nishiura H. Reduced risk of importing Ebola virus disease because of travel restrictions in 2014: a retrospective epidemiological modeling study. PLoS ONE 11(9), e0163418 (2016).

7 Shaman J, Kohn M. Absolute humidity modulates influenza survival, transmission, and seasonality. Proc. Natl Acad. Sci. USA 106(9), 3243-3248 (2009).

8 Nishiura H, Tsuzuki S, Yuan B et al. Transmission dynamics of cholera in Yemen, 2017: a real time forecasting. Theor. Biol. Med. Model. 14(1), 14 (2017).

9 Dinh L, Chowell G, Mizumoto K et al. Estimating the subcritical transmissibility of the Zika outbreak in the State of Florida, USA, 2016. Theor. Biol. Med. Model. 13(1), 20 (2016).

10 Nishiura H. Real-time forecasting of an epidemic using a discrete time stochastic model: a case study of pandemic influenza (H1N1-2009). Biomed. Eng. Online 10, 15 (2011). 